\title{
A WEB AGENT FOR CONCEPTUAL COST ESTIMATION OF HIGHWAY CONSTRUCTION PROJECTS
}

\author{
Wen-der Yu and Jia-wei Fu \\ Institute of Construction Management, Chung Hua University, Taiwan
}

\begin{abstract}
Accurate cost estimation in the early stage of project lifecycle is essential for both effective financial planning and cost control of construction projects. Previous efforts by the research team have developed a Web-based Intelligent Cost Estimator (WICE) system. This paper describes a further step of WICE research to develop a special-purpose web agent for various highway construction project participants to utilize WICE. The proposed system has shown improvement on the usability of WICE so that even users without estimation knowledge can utilize the functions provided by WICE.
\end{abstract}

Keywords: Web agent; Neuro-fuzzy; Conceptual estimation; Highway construction; WICE.

\section{INTRODUCTION}

The cost estimation in conceptual planning phase provides the foundation for both effective financial planning and cost control of construction projects. However, accurate cost estimation is hard to obtain before the detail design due to the complete design information is not available at that stage. In order to improve this problem, many conceptual cost estimation methods have been proposed including statistics based methods (e.g., unit-area method, unit-volume method, unit-capacity method, etc.), principal items method (e.g., principal material items method and principal activities method), cost index method, and parametric estimation method [1]. Among the above conceptual cost estimation methods, parametric estimation method has been proved capable of generating accurate estimates comparable to the results with detail estimation method [2,3]. As a result, the research team developed a Web-based Intelligent Cost Estimator (WICE) [4] to meet the needs of the construction industry.

The WICE system is based on a previously proposed PIREM (Principal Items Ratio Estimation Method) [1], which combines the Principal Items Method and Parametric Estimation Method to form a new conceptual cost estimation method. Another feature of WICE is the integration of a neuro-fuzzy artificial intelligence technique and the web based information technology to provide a web-based intelligent cost estimation system. The WICE system provides many desirable features for construction cost estimation such as real-time estimation function, sensitivity analysis function, low maintenance cost, global accessibility, and high estimation accuracy. However, the WICE requires users to input values of influential attributes for various types of construction projects. Such attribute values are based on the "engineering judgments" of the domain experts (or experienced engineers). Such requirements have put limitations on the usability of WICE system, since in most cases the decisions during feasibility analysis stage are made by top managers or investors who are not "domain experts" for cost estimation. Moreover, for public construction projects, the budgets should be reviewed and audited by the county councilors, the state assemblymen, or the legislators of the state who are not specialized in construction cost estimation. A need rises to develop a system that can assist the users of WICE in entering attribute values and organizing estimation results, so that users without engineering background can also enjoy the functions provided by WICE.

The paper is presented in the following manner. Literature related to web agents is reviewed first. Then, the framework and functions of WICE system is described in details. In the fourth section, the web agent for highway construction cost estimation is proposed. The system is implemented and testing in Section Five. Finally, conclusions and future works are discussed at the end of this paper.

\section{REVIEW OF WEB AGENT}

The web agent is an emerging information technology that incorporates World Wide Web (WWW), databases, and artificial intelligence (AI) technologies. This section reviews some literature related to web agent.

\subsection{Definition of Web Agent}

Nwana proposed three characteristics required for an agent [5]: (1) autonomy-meaning that an agent should act independently in performing their functions without requirement of human guidance; (2) cooperation - the agent should be able to cooperate and communicate with human users or other agents; (3) Learning - the agents should be able to learn 
through the interaction with users to improve their performance.

Wooldridge and Jennings [6] defined "intelligent agents" as computer systems that act adaptively and independently to achieve their goals. Sycara [7] gave definition of "intelligent agents" as "a computer system that assistant the human users in time consuming information collection, information searching, and integration of fragmented information", and. Bradshaw considered agent as a system that represents the user to communicate with other system.

Harrison et al. believed that an intelligent agent has the following features: (1) delegation-the functions of agents are determined by the user; (2) communication-the agent should be able to communicate effectively the user; autonomy-even though the functions of agents are determined by the user, the agent should be able to complete its job without directions provide by human users; (4) monitoring - the agent should be able to sense the influence of environmental changes, so that outputs of the system adapt accordingly; (5) actuation - the agent should be able to act according to its reasoning result; (6) intelligence-agent should possesses some sort of knowledge that might be stored in forms of decision rules, patterns, and predetermined procedures; (7) Others.

Bryson et al. defined the term agent as any autonomous actor with sets of: (1) goals - conditions the agent works to achieve or fulfill; (2) intentions - goals and sub-goals the agent is currently engaged in pursuing; (3) beliefs - knowledge about the world (which is limited and possibly inaccurate); and (4) behaviors - actions the agent is able to take.

\subsection{Review on Web Services}

Web services are application programs that users can access and execute via internet without the installation on the client's platform [9]. Thus, the web services basically perform the same functions as PC-based programs. In contrast to the PC-based programs, the web service program does not execute on the client's PC, but on the server. Different from web pages, web services are not merely an information bulletin that provides static information. It interacts with the users and even execute programs including database searching and other computational programs, so that complicated functions can be performed by the web services programs.

Some merits of web services include $[4,10]$ : (1) centralized databases and knowledge bases management - update of database and knowledge base are guaranteed; (2) minimum requirement on client's platform - many application programs require platform system to provide sufficient hardware capacity and capability, web services place hardware and software requirements only on the server, thus save costs for the clients; (3) globally and all-time accessible — web services are opened $24 \mathrm{hrs}$ everyday, users around the world can access the application anytime and anywhere; (4) minimized maintenance costs - the centralized databases and single server system can lower the system maintenance costs.

In spite of the many merits described above, web services also hold several drawbacks, such as (1) requirement of internet connection - the quality and safety of the program execution are relied on the quality and safety of internet connection; (2) security of client's information-many web services require the users to input information of related attributes of the program and even personal information, this may cause security problems of the users' information; (3) requirements on formatted inputs - unlike other web searching engines, the web services require users to input information as the web interface requires, thus the flexibility of requests by the users are limited; (4) system stabilization - the centralized platform may cause problem of stabilization, this can be mediated by back up systems.

\section{WEB-BASED INTELLIGENT COST ESTIMATOR (WICE)}

In order to meet the requirement of real-time cost estimation, an intelligent web based cost estimation system namely Web-based Intelligent Cost Estimator (WICE) was developed under the collaboration efforts of Chung Hua University and the industrial partner, CHINA ENGINEERING CONSULTANTS, INC. (CECI), Taipei, Taiwan. This section describes the WICE system and its underlying theories in details to provide backgrounds for the development of web agent in this paper.

\subsection{Theoretical Backgrounds of WICE}

(1) Principal Items Ratio Cost Estimation Method (PIREM) - the essential problem for all analog based cost estimating approaches is that the unit prices of cost items fluctuate as time passes. Thus, construction cost estimates obtained based on previous estimation experiences may be incorrect due to unit price variation in the marketplace. The key problem is the use of "activity cost" as the measure of estimates during analogizing process. In WICE a Principal Items Ratio Cost Estimation Method (PIREM) was adopted to take the "unit price" and "activity quantity" apart in an activity cost item. Previous knowledge acquired from historical data is used to estimate the quantities of an activity. On the other hand, the most updated unit price information is surveyed in the marketplace to reflect the current unit prices of material, labor, and equipment.

In order to simplify and reduce the estimation effort, only the most important cost items are taking into account for cost estimation. Thus, the Pareto Optimum 
Criterion (or named "80/20 Principle") is adopted. Simply stated, the Pareto Optimum Criterion suggests that $80 \%$ of the overall project cost is determined by $20 \%$ of the cost items [11]. Therefore, instead of estimating the quantities of all cost items, only the top $20 \%$ most important cost items' quantities are estimated and their related unit prices are inquired. The selected top $20 \%$ cost items is called "principal items $(P I)$ ". The summation of quantities and unit prices of the principal cost items constitutes the cost of principal items $(P I C)$. The ratio of $P I C$ over the overall cost is defined as the principal item ratio (PIR or $p$ ). The value of $p$ can be calculated by the following equation.

$$
p^{s}=\frac{\sum_{j=1}^{l} U P_{j}^{s} \cdot Q_{j}^{s}}{\sum_{i=1}^{n} U P_{i}^{s} \cdot Q_{i}^{s}}=\frac{P I C^{s}}{O C^{s}},
$$

where the numerator is the summation of the costs of all principal items, while the denominator is the summation of the costs of all cost items in a project. The super script $s$ upon all parameters stands for the $s^{\text {th }}$ historical example. $U P_{i}$ means unit price and $Q_{i}$ means quantity of the $i^{\text {th }}$ cost item, and so forth. $O C$ stands for overall cost. The ratio $p$ obtained in Eq. (1) is call "principal item ratio $(P I R)$ " as defined above.

It is found by analyzing PIR's obtained from historical cost estimation data that the PIR of a specific type of construction project usually keeps constant with very small variation [1]. Therefore, given the $P I C$, the $O C$ of the new project can be recovered by the following equation.

$$
O C^{r}=\frac{P I C^{r}}{\bar{p}},
$$

where $O C^{r}$ is the estimate of overall cost for the new $\left(r^{\text {th }}\right)$ project. $\bar{p}$ is the average PIR calculated from previous projects. $P I C^{r}$ is the cost of principal items of the new $\left(r^{\text {th }}\right)$ project.

In Eq. (1) and (2), the current unit prices (UP's) for the new project should be inquired from the marketplace at the moment of estimation. While, the quantity, $Q$, and average PIR, $\bar{p}$, have to be determined by parametric estimating methods based on the pre-determined mathematic relationships. This relationship should be established according to the knowledge acquired from historical data.

(2) ANFIS neuro-fuzzy data mining - in order to establish the relationship between influential attributes and activity quantities, a neuro-fuzzy data mining technique named ANFIS [12] was adopted to mine estimation knowledge from historical data. Figure 1 shows the architecture of ANFIS network, where the network consists of 6 layers: (1) Layer 0 - input layer; (2) Layer 1-membership functions; (3) Layer 2-t-norm operation; (4) Layer 3-normalization of inference output; (5) Layer 4 Sugeno FIS (fuzzy inference system) operation; and (6) Layer 5-aggregation operation for output.

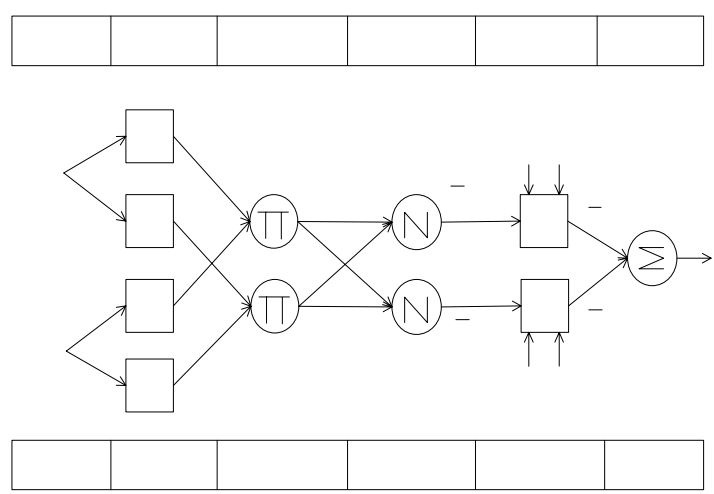

Figure 1. ANFIS network architecture [12]

The Sugeno FIS operation adopts a linear function in the consequence of a fuzzy decision rule, so that the defuzzification of FIS becomes straightforward. From extensive application testing results, it was found that ANFIS is extraordinarily excellent in mining nonlinear relationships existing in the historical databases [2].

\subsection{Framework of WICE}

The Matlab 6.3 with Web Server and Fuzzy Logic Toolboxes is adopted for WICE development. The platform selected for WICE implementation consists of: (1) PC CPU: Pentium IV 1.5G; (2) OS: Windows 2000 Server; (3) SRAM: 1.0 G. Since the ANFIS trainings can be done offline, the CPU speed for the web server may be not demanding. However, broader bandwidth for transmission is required if the number of simultaneous accesses or need on graphic transmissions is large. The framework and data flows of WICE are depicted in Figure 2, where the Client (User) $A$ submits parameter inputs (including influential attributes and unit prices) and job request to WICE by filling out the Parameter Input Sheet. The WICE then transmits the input data to Matlab main system via the interface and web-server programs. The Matlab main system consults related Matlab programs and performs the required calculations (including ANFIS network mapping), and then the results (including estimation results and relevant graphics) are generated. The numeric data (resulted estimates) and graphics are handled 
separately hereafter. The numeric data are transmitted backward via web-server and interface programs to the respond form of the Client. In the meanwhile, generated graphics (if applicable) are loaded on the respond form. Thus the request of Client $A$ is completely served. Multiple requests by different users are served simultaneously via the similar process for Client $A$ described above, while buffer of memory (i.e., capacity of RAM) for storing intermediate data is required as the number of simultaneous users increases. The intermediate data are handled by the web server and interface programs so that no conflict occurs while multiple users are accessing WICE.

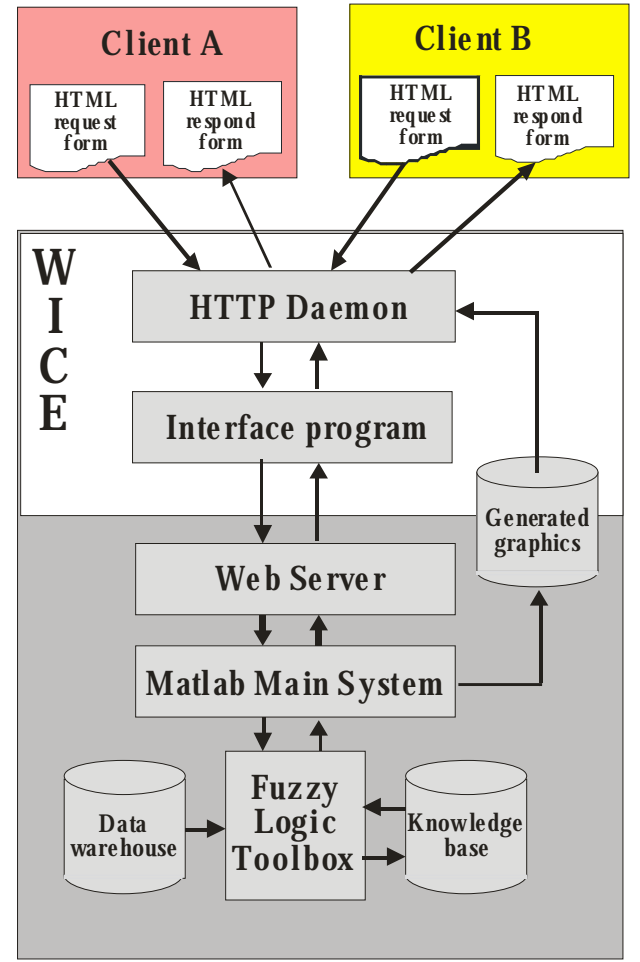

Figure 2. Framework and data flows of WICE [4]

\section{PROPOSED WEB AGENT FOR HIGHWAY CONSTRUCTION COST ESTIMATION}

This section proposes a web agent for highway construction cost estimation based on the WICE system described in the previous section.

\subsection{Highway Construction Cost Estimation Process}

The cost estimation process for highway construction projects using WICE system is studied by interviews with domain experts of CECI [2]. The resulted process is depicted in Figure 3. The process consists of three major steps: (1) collecting basic information regarding to the influential attributes based on the SPEC's and drawings; (2) estimating the values of influential attributes based on the judgments of domain experts or applying expert rules; (3) entering the values of influential attributes into
WICE system and presenting the estimation results.

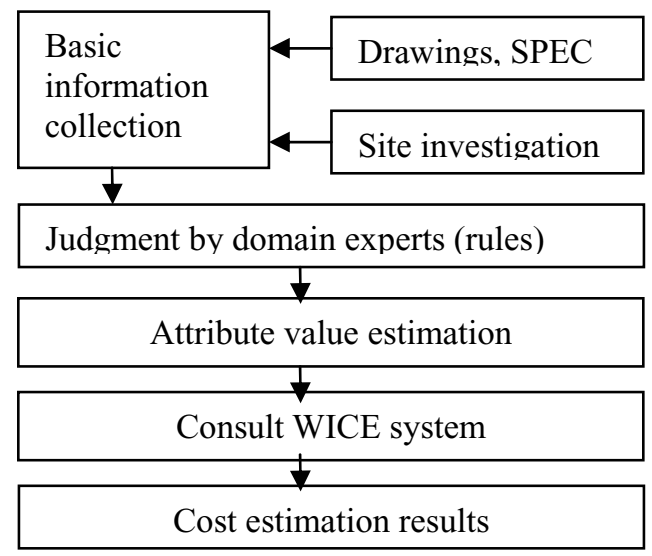

Figure 3. Process of highway construction cost estimation with WICE

4.2 Knowledge Base of Web Agent

The key for the "intelligence" of the web agent is the underlying knowledge stored in the knowledge base. In this case, the knowledge of the domain experts is stored in terms of IF-THEN rules. In this research, totally 65 IF-THEN rues were generated through interviews with experiences estimators. These rules are stored in the form of decision tree. Figure 4 is a part of the such decision tree.

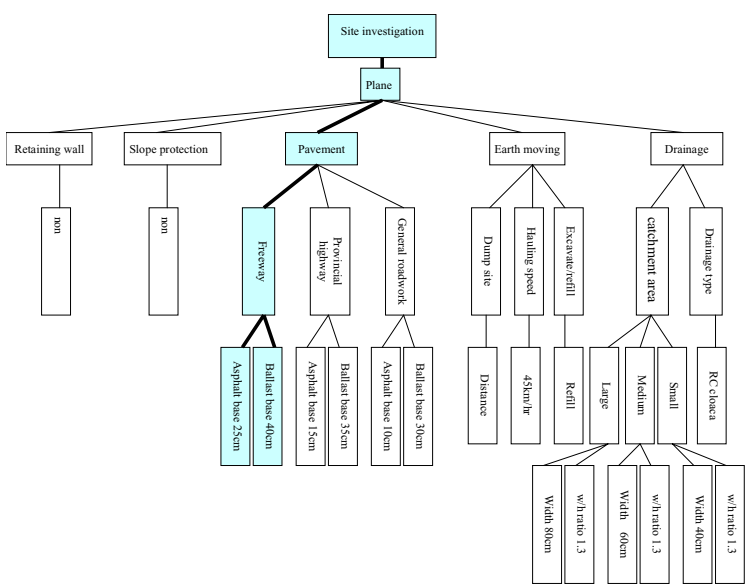

Figure 4. Part of the decision tree for IF-THEN rules

The shadowed boxes show two examples of such IF-THEN rules:

Rule \#1:

"IF Site is Plane and Class is Freeway,

THEN Asphalt is $25 \mathrm{~cm}$ for Pavement". Rule \#2:

"IF Site is Plane and Class is Freeway,

THEN Ballast Base is $40 \mathrm{~cm}$ for Pavement".

In the "IF-part" of the example rule, the underlined terms are names of factors that affect the influential attributes, the italic terms are values of the such factors. In the "THEN-part", the underlined terms are names of the influential attributes; the italic terms are values of the attributes that are generated by the rules. 


\subsection{Framework of Web Agent}

Framework of the proposed web agent system is shown in Figure 5. The usage process of the proposed system consists of 6 steps: (1) assessment of attributes - by the process shown in Figure 3; (2) collaboration with various agents in WICE- to generate estimation results for each activities; (3) presentation of the estimation results to user; (4) evaluation of the estimation results by domain expert-if the results are approved, the estimation results are accepted and stored in the historical database as a new data, otherwise are expelled; (5) training of knowledge base - after a period of time, the knowledge base is updated by system training; (6) update of knowledge base of each agent.

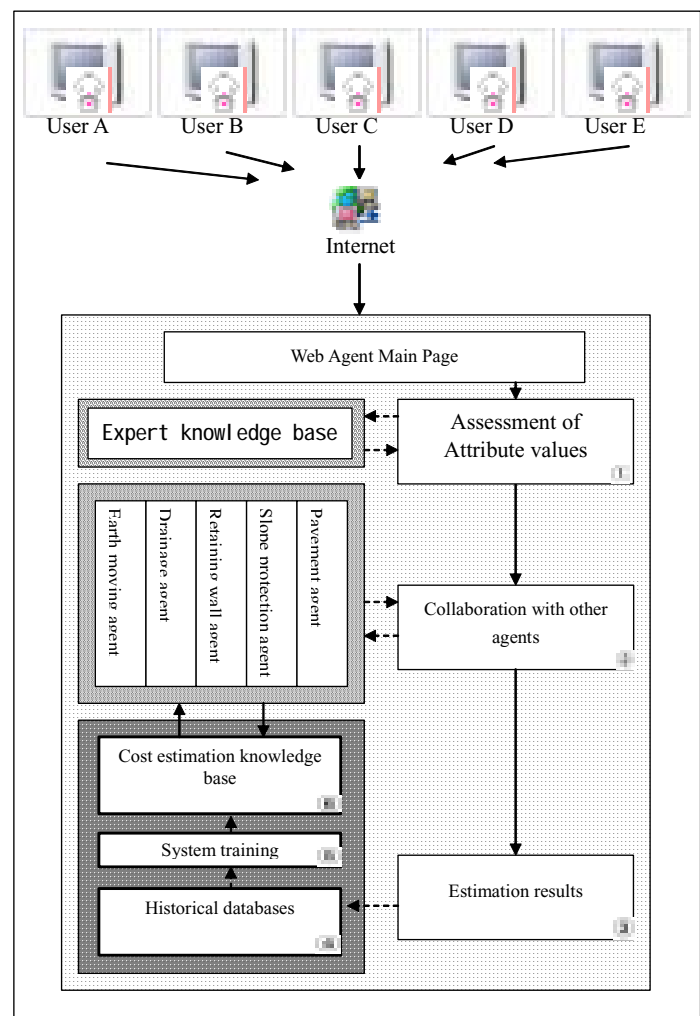

Figure 5. System framework of proposed web agent

\section{SYSTEM IMPLEMENTATION AND TESTING}

The proposed web agent for WICE system to facilitate various users of highway construction project participants is implemented as a web interface that dynamically interact with the users and WICE system to acquire required information for conceptual cost estimation, and provide collective information of estimation results.

The implemented system is shown in Figure 6 9. The primitive version of the proposed system is developed in Chinese language. Figure 6 shows the main page of the proposed web agent. Figure 7 shows the web page acquiring basic information from the user. In Figure 7, the basic information is in forms of questions of common sense, so that users who are not experienced estimators can also answer the questions. Should the user be not able to answer the question, the system provides default "best guess" solution of the user.

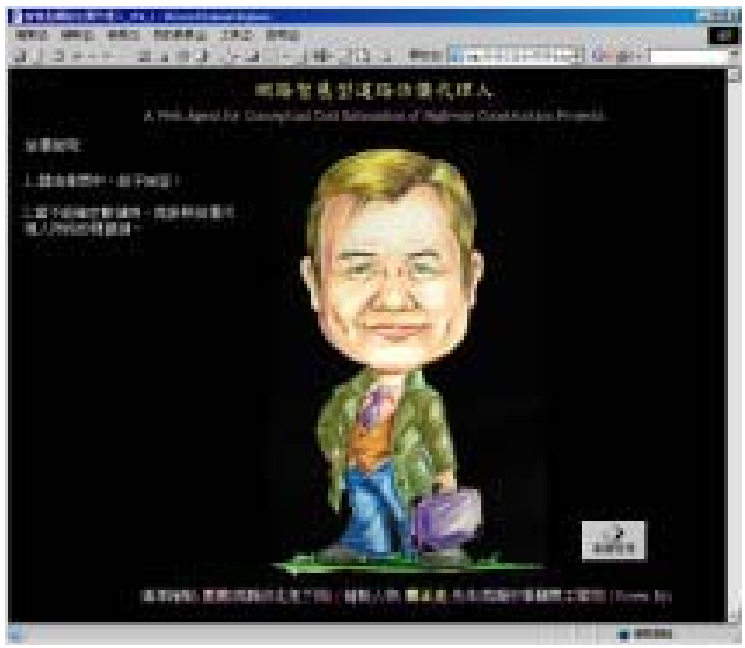

Figure 6. Main page of proposed web agent

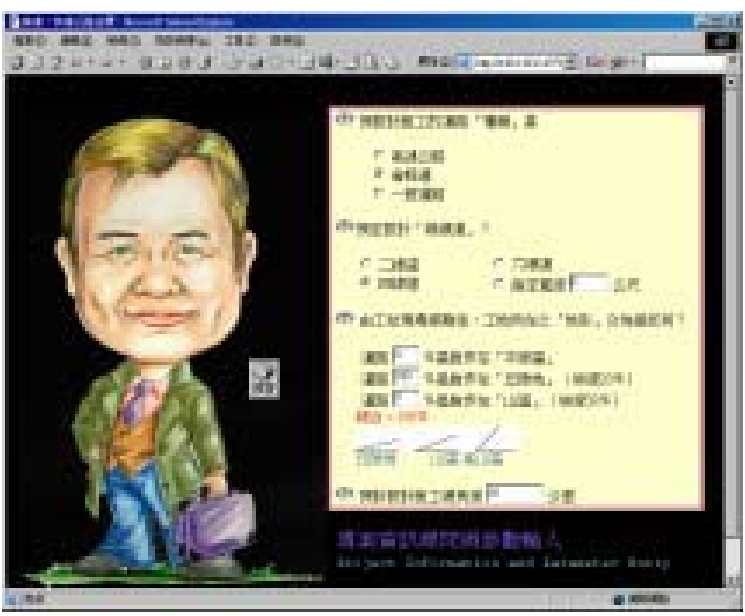

Figure 7. Web page for basic information collection

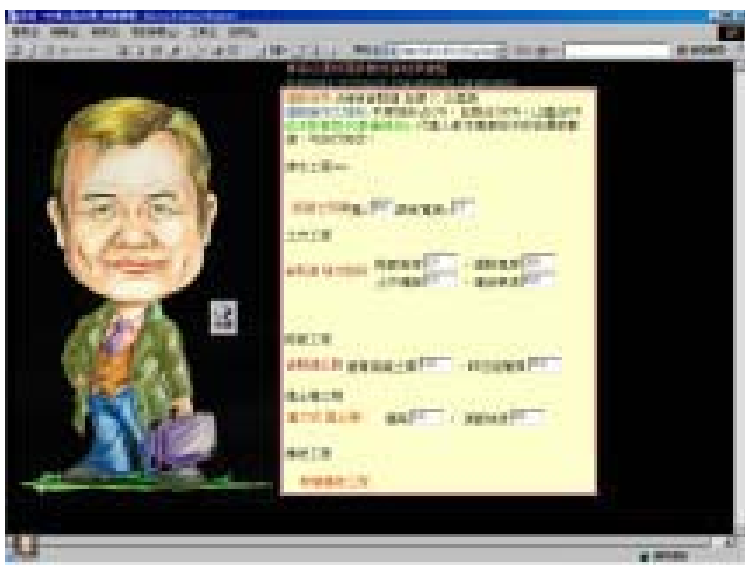

Figure 8 . Attribute values estimated by expert rules

Figure 8 shows the results of the estimated attribute values obtained by the system after applying expert rules. Up to this stage, the required input 
attribute values are generated. In Figure 8, the user is allowed to fine-tune the estimated values by directly entering his/her values.

The final step is to generate the overall estimation results by collaboration of various agents of WICE, including Earth moving agent, Drainage agent, Retaining wall agent, Slope protection agent, and Pavement agent. The attribute values are passed internally in WICE to the above mentioned agents. The estimation results of each agent are generated and shown in Figure 9. The overall project cost estimation is also shown on the top in Figure 9 to provide final estimation result for the user.

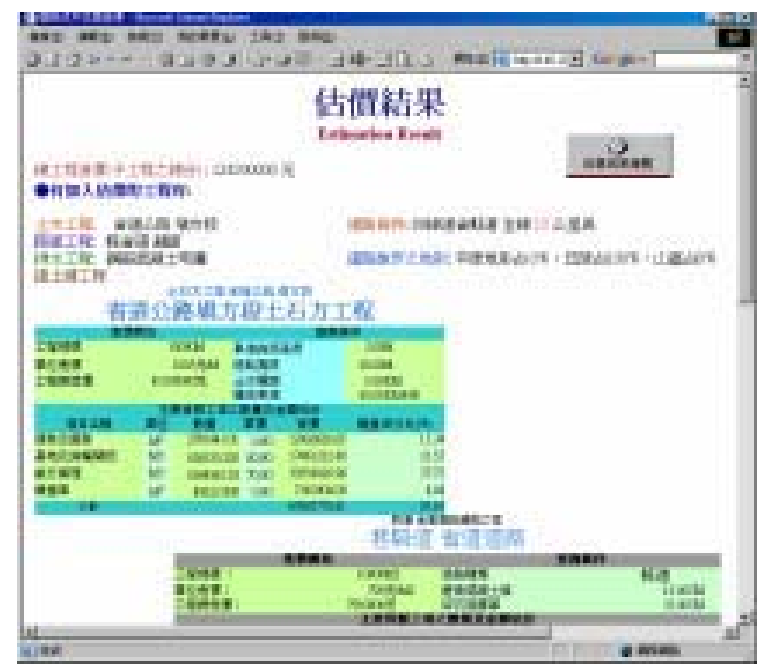

Figure 9. Presentation of estimation results

With aids of the proposed web agent, even inexperienced users can fully exploit functions provided by WICE. Real world examples of highway construction projects have been tested in the implemented web agent to verify the system. Due to the limitation of the paper, the testing process is not shown here. However, the results show that the proposed system can generate identical results as the domain experts do using WICE.

\section{CONCLUSIONS AND FUTURE WORKS}

This paper presents a web agent for cost estimation of highway construction projects. The proposed system is an extension of previous research-Web-based Intelligent Estimator (WICE). The proposed system integrates WICE with the knowledge of domain experts to provide users, who are not experienced cost estimators, an assistant for using WICE system. The proposed system is implemented and tested with real world example projects. It is found that the proposed system can generate cost estimation result similar to the domain experts while using WICE system.

Future directions after this research include (1) automated learning process of expert knowledge base for acquiring information provided by the users; (2) application of same methodology to build agent for other type of works; (3) more on the domain knowledge acquisition that is not fully researched in this paper.

\section{REFERENCES}

[1] W. D. Yu, and J. B. Yang, Final Report on the Development of a Neuro-Fuzzy Knowledge-based System for Construction Conceptual Estimation, CECI, Taipei, Taiwan, 2002. (in Chinese)

[2] W. D. Yu, and J. B. Yang, "Data Mining for the Cost Estimating of Highway Bridges Construction with a Neuro-Fuzzy System", Proceedings of 2001 Ninth National Conference on Fuzzy Theory and Its Applications, Nov. 23 24, National Central University, Chung-li, Taiwan, 2001, pp. 437 442.

[3] Wilmot, C. G., and Cheng, G., "Estimating Future Highway Construction Costs," Journal of Construction Engineering and Management, Vol. 129, No. 3, pp.272-279, 2002.

[4] Yu, W. D., and Lai, C. C., "WICE: A Web-based Intelligent Cost Estimator for Real-time Decision Support," Proceedings of IEEE/WIC International Conference on Web Intelligence (WI'03), Oct. 13 16, Session 10B, October 13-16, 2003, Lord Nelson Hotel, Halifax, Canada, pp. 646 649, 2003.

[5] Nwana, H.S., "Software Agents: An Overview," Knowledge Engineering Review, Nol.11, No.3, pp. 205-244, 1996.

[6] Wooldridge, M., and Jennings, N. R., "Intelligent Agents: Theory and Practice", The Knowledge Engineering Review, Vol. 10, 1995.

[7] Sycara, K. A., Williamson, P. M. and Zeng, D., "Distributed Intelligent Agent”, IEEE Expert, 1996.

[8] Bryson, J. J., Martin, D., McIlraith, S. A., ad Stein, L. A., "Agent-based composite services in DAML-S: the Beavior-oriented design of an intelligent semantic web," article in Web Intelligence, edited by Zhong, N., Liu J., and Yao, Y., Springer, Berlin, Germany, pp. 37-58, 2003.

[9] Wang, Y. S., "Application of web service technologies in construction information system," Master Thesis, Department of Civil Engineering, National Taiwan University, Taiwan, R.O.C., 2004. (in Chinese)

[10] Pride, W. M., and Ferrell, O. C., Marketing: Concepts and Strategies, 12 edition, Houghton Mifflin Company, NY, USA, 2003.

[11] R. Koch, The 80/20 Principle-The secret of achieving more less, Nicholas Brealey Publishing Ltd., London, 1997.

[12] Jang, J. S., "ANFIS: Adaptive-network-based fuzzy inference system," IEEE Trans. on Systems, Man, and Cybernetics, Vol. 23, No. 3, 1993, pp. 665-685. 